\title{
La dualité du temps dans le théâtre d'Armand Salacrou
}

Dr. Marguerite Mimi Maurice

Maître de conférences au département de

Français à la Faculté de Pédagogie d'Ain Shams 



\section{Résumé}

Armand Salacrou, reconnu en France comme l'un des écrivains marquants de sa génération et précurseur d'une révolution scénique radicale qui a initié le plus systématiquement à disloquer les vieilles formes dramatiques et dont nous voyons aujourd'hui les effets. L'Inconnue d'Arras est une pièce quasi cinématographique, et l'idée d'un personnage revoyant toute sa vie dans la seconde où il meurt, n'a pas du tout vieilli. Salacrou est allé jusqu'à menacer son temps et le monde du théâtre en exposant des procédés temporels qui s'apprêtaient à bouleverser pour toujours la voie spatio-temporelle de la scénographie. Parmi ces aspects visant le renouvèlement des formes dramatiques en vigueur et la mutation de l'architecture dramatique traditionnelle, en usant de la dimension temporelle, le retour en arrière dans L'Inconnue d'Arras, l'appel à une inversion complète du temps dans Sens interdit ou à des chevauchements de la durée comme dans L'Archipel Lenoir où Salacrou a renversé la chronologie rituelle. Ces remaniements du temps, toujours annoncés par une note du dramaturge, sont en réalité des nouveautés pour le théâtre (et même pour le cinéma) qui jusqu'à 1929 ne connaissait ni "flash-back", ni "jeux sur le temps". 
Au fait, l'œuvre d'Armand Salacrou reflète une certaine soif de la fuite du temps et surtout une tentative angoissée de trouver un sens à la vie. Il a traité le thème de la temporalité avec sa dextérité habituelle, sa grande maîtrise de la scène et de la langue, sa verve mordante et satirique.

Mots-clés : Théâtre ; temps ; Salacrou ; Dualité

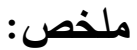

أرماند سالاكرو ، المعترف به في فرنسا كواحد من الكتاب البارزين من جيله ونذير ثورة مرحلة جذربة أدت بثكل منهجي إلى تعطيل الأثكال الدرامية القديمة والتي نراها اليوم. كل مسرحيات سالاكرو سينمائية تقريبًا ، وفكرة شخصياته التى تستعرض كل حياته في الثانية التي يموت فيها و لم تتقدم في السن على الإطلاق. ذهب سالاكرو إلى حد تهديد عصره وعالم المسرح من خلال عرض عمليات زمنية كانت على وشك أن تزعج إلى الأبد المسار المكاني والزماني للسينوغرافيا. من بين هذه الجوانب التي تهذف إلى تجديد الأشكال الدرامية السارية وتحوير العمارة الارامية التقليدية ، من خلال الاستفادة من البعد الزمني ، والارتجاع ، واللدعوة إلى انعكاس كامل للوقت في المعنى أو التداخل في المدة حيث عكس سالاكرو التسلسل الزمني للطقوس. هذه التعديلات في الوقت ، التي يتم الإعلان عنها دائمًا من خلال ملاحظة من الكاتب المسرحي ، هي في الواقع جديدة على المسرح (وحتى بالنسبة للسينما) التي لم تكن تعرف حتى عام 1929 "الفلاش باك" ولا "الألعاب في الوقت

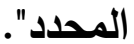
في الواقع ، يعكس عمل أرماند سالاكرو تعطثًا معينًا لرحلة الزمن وقبل كل شيء محاولة بائسة لإيجاد معنى للحياة. لقد عالج موضوع الزمنية ببراعته المعتادة ، إتقانه الكبير للمرحلة واللغة ، حيويته اللاذعة والساخرة. 
على الرغم من الوقت "الذي تم لعبه" بأثر رجعي قبل الممثلين ومن قبلهم ، إلا أن سالاكرو يصر على استحالة التواصل الفعال مع الماضي. طور أسلوبًا مشابهًا لموضوع الاغتراب الزمني الأي يعمل على إتقان تقنياته السينوغرافية ، أي ذكريات الماضي والتسلسلات الزمنية الصغيرة.

"Si le théâtre est irremplaçablement à la fois plaisir et savoir c'est qu'il lui est demandé à chaque moment de saisir ce qui sépare ce moment de l'instant qui l'a précédé, de comprendre du même coup le pourquoi du changement... "

\section{Anne Ubersfeld ${ }^{1}$}

Armand Salacrou, I'un des plus grands dramaturges français du $\mathrm{XX}$ siècle, a choisi le théâtre comme moyen de fuir son ennemi redoutable qui est le temps, et ce en créant d'autres vies, en « diluant son destin dans un jeu de destinées inventées.» ${ }^{2}$ Entre le temps vécu et le temps imaginé, le temps présent et le temps passé, les personnages salacriens sont indélébilement pris dans un cercle vicieux ; tiraillés entre finitude et infinitude, ils gèrent leur existence en tenant compte de la dualité illimitée du temps.

${ }^{1}$ Anne, UBERSFELD, Galions engloutis, textes réunis par Pierre Frantz, Isabelle Moindrot et Florence Naugrette, avant-propos de Michel Vinaver et de Jean-Marie Villégier, Presses Universitaires de Rennes, collection «Le Spectaculaire », 2011, p. 21.

${ }^{2}$ Armand, SALACROU, Les idées de la Nuit, Librairie Arthème Fayard, Paris, 1960, p.9. 
Pourtant, le dramaturge, qui considère le Temps comme maître absolu de ce monde, a réussi à travers ses pièces à contourner cette triste emprise. Reconnu en France comme le précurseur d'une révolution scénique radicale qui a initié le plus systématiquement à disloquer les vieilles formes dramatiques, révolution dont nous constatons les effets jusqu'à nos jours, il écrit en 1935 L'Inconnue d'Arras, une pièce quasi cinématographique où l'on découvre un personnage qui vient de mourir et qui est condamné à revoir toute sa vie passée dans une sorte de flash-back réitéré. Sans être considérée par la critique comme l'une des grandes œuvres dramatiques du XXème siècle, elle mérite d'être lue avec attention car elle recèle des idées assez surprenantes, curieusement modernes à cette époque notamment celle de la reprise de la vie après la mort, thème qui sera ultérieurement reproduit par d'autres écrivains dont le grand Jean-Paul SARTRE avec ses Jeux sont faits. De façon générale, nous pouvons affirmer que Salacrou est allé jusqu'à menacer son temps et le monde du théâtre en exposant des procédés temporels qui s'apprêtaient à bouleverser pour toujours la voie spatio-temporelle de la scénographie.

Parmi ces aspects visant le renouvellement des formes dramatiques en vigueur et la mutation de l'architecture dramatique traditionnelle en usant de la dimension temporelle : citons le

\section{4}


retour en arrière dans $L$ 'Inconnue d'Arras, l'appel à une inversion complète du temps dans Sens interdit ou les chevauchements de la durée comme dans L'Archipel Lenoir où Salacrou a renversé la chronologie rituelle. La dualité du temps, toujours annoncée par une note du dramaturge, est en réalité une nouveauté pour le théâtre (et même pour le cinéma) qui jusqu'à 1929 ne connaissait ni "flash-back", ni "jeux sur le temps".

$\mathrm{Au}$ fait, l'œuvre d'Armand Salacrou reflète une certaine soif de l'unification du temps et surtout une tentative angoissée et vaine de trouver un sens à la vie. Le dramaturge a traité le thème de la temporalité avec sa dextérité habituelle, sa grande maîtrise de la scène, de la langue et sa verve mordante et satirique.

"Prisonnier du ciel, l'homme traîne la littérature à sa remorque $»^{3}$. Mais notre dramaturge, lui, se sentait prisonnier, non pas du ciel, comme il l'avait écrit pour présenter sa première œuvre imprimée, Le Casseur d'assiettes, mais plutôt du temps. Une inquiétude, qui semble témoigner d'une prise de conscience du mal-être, va l'accompagner tout au long de sa vie, le tourmenter et se traduire dans ses œuvres.

Les personnages salacriens contribuent à cette dualité du temps à travers leur double fonction. Ils cherchent une identité et une 
raison pour leur existence, tout en exprimant leur angoisse face au temps qui leur échappe.

Les pièces de Salacrou « témoignent de la déconstruction de l'art dramatique où les piliers séculaires du théâtre sont menacés par le rejet du principe de déterminisme et par la mise en cause des lois physiques, tout comme les notions du temps et de l'espace ${ }^{4}$.

C'est ainsi qu'elles marquent indéniablement un pas vers le théâtre onirique. Le modèle du théâtre de Salacrou se répand donc sur les scènes françaises alors que les premières contestations du " théâtre service public » ${ }^{5}$ se font entendre.

Ce théâtre, dit Henri-René Lenormand, est "Celui où le rêve, sous toutes ses formes, prend le pas sur le réel, [...] se détache sans équivoque possible de ce que le théâtre nous donne pour réel, puisque à la scène, c'est déjà de la réalité transposée et rêvée "6.

${ }^{4}$ Edwige, GBOUABLE, des écritures de la violence dans les dramaturgies contemporaines d'Afrique noire francophone, (1930-2005). Littératures. Université Rennes 2, 2007, p. 24. ${ }^{5}$ Léa VALETTE, Histoire des arts et des représentations. Les lieux de la critique de théâtre en France : Enjeux esthétiques et convictions politiques, thèse, Université Paris-Ouest Nanterre, novembre 2014, p.1068.

${ }^{6}$ Henri-René LENORMAND, Les Confessions d'un auteur dramatique 2, Paris, Albin Michel, 1953, p. 68. 
$\mathrm{Au}$ fait, les conceptions insolites de notre dramaturge permettent de faire embrouiller le temps. "La seconde qui s'enfuit a dit ce que la seconde qui suit ne pourra pas dire.» ${ }^{7}$

Pour évoquer la dualité du temps dans son théâtre, Salacrou se montre perdu entre son passé et son avenir. Son passé est inchangeable, son présent n'est pas à maîtriser, même s'il paraît à sa portée, et son futur est imprévisible. Toutes ces conditions augmentent le sentiment d'impuissance chez le personnage face au temps.

Les notions du temps de la narration et du temps narré correspondent à l'idée que Georges de la pièce Une Femme Trop Honnête affirme : «C'est le futur qui donne un sens au passé, le présent est aveugle ${ }^{8}$. De même Lucie, de la pièce Miroir, se plaint sans cesse de son passé irrévocable et morose : «Nous sommes condamnés à être celui que nous avons été. A quoi bon expliquer le passé ? Dieu est moins puissant sur les hommes que leur propre passé, définitif. Ce qui est fait, est fait pour /'éternité.»"

${ }^{7}$ Anne UBERSFELD, op.cit. p.23.

${ }^{8}$ Armand SALACROU, Une Femme trop Honnête, Paris, Gallimard, collection Folio, 1956, p.19.

${ }^{9}$ Armand SALACROU, Le Miroir, in Théâtre VII, Paris, Gallimard, Nrf, 1956, p. 206. 
À son tour, Salacrou, à l'image de ses personnages, résume son propre conflit avec le temps: "Dans mon angoisse, c'est l'arrivée du futur qui m'apparait. Que vais-je devenir ? Tomber? M'accrocher? Et dans cette angoisse, c'est le passage du futur inéluctable vers un passé ineffaçable, qui m'étreint. Dans l'anxiété, je ne suis que le témoin de ma vie. " ${ }^{10}$

Le futur est ainsi aussi dur à supporter que le passé car, non seulement il pèse sur le moral des personnages mais il les humilie et les terrifie avec les surprises fâcheuses qu'il pourrait apporter. Cette notion de la dualité du temps n'était donc pas d'usage avant Salacrou au théâtre qui renvoie au discours narratif fixant une temporalité précise. II s'agit ainsi de diverses séries de temps fictif et variable en relation fonctionnelle avec les différentes étapes de l'action, de façon à ce que le protagoniste salacrien puisse résumer ce qui s'est passé pendant de longues années en une simple action ou effet de mime en quelques minutes. Le dramaturge s'intéresse aussi à l'intériorisation des événements en les retirant de la réalité et les dégradant de tous les indices spatio-temporels. Et les personnages restent ainsi plongés dans la rêverie, perdus entre le passé et l'avenir, dans un temps irréel.

\footnotetext{
${ }^{10}$ Armand SALACROU, « Mes certitudes et incertitudes » in théâtre VI, Paris, Gallimard, nrf, 1954, pp.212-213.
}

\section{8}


Salacrou a tenté «l'expression de l'inconscient, d'où sa quête éperdue de l'espace intérieur" dans lequel prennent naissance les forces, l'espace et le temps sujets à modifications ». ${ }^{11} \mathrm{C}$ 'est ainsi que l'action dramatique dans L'Inconnue d'Arras se limite à un retour en arrière mettant en scène tous les moments de la vie du protagoniste qui vient de se donner la mort. La durée de l'intrigue se restreint entre le moment de déclenchement du coup de revolver et le décès du personnage, une fraction de seconde que le dramaturge étale ingénieusement en trois actes. C'est alors que le temps se fige ou semble ne plus subsister et tout ce qui se passe est toujours traduit par l'intermédiaire des âmes déchirées des protagonistes.

Au fait, le temps rétrospectif qu'Ulysse revit n'est pas "raconté" comme à l'accoutumée mais "joué", ce qui mène les spectateurs à une autre dimension scénographique. Tous les acteurs de la vie d'Ulysse sont interpelés pour paraître directement et physiquement sur scène incarnant la même personne en deux périodes et états différents, jeunesse et vieillesse, en vie et trépassée. Pour accentuer l'idée de la dualité du temps, Salacrou a insisté à mettre en considération pendant la mise en scène de 
la pièce, les détails suivants : le jeu du clair-obscur, les ombres, les différents rejets de lumière qui présentent et insistent sur l'idée de la coexistence des morts et des vivants, le temps rétrospectif et le temps actif qui cohabitent sur scène, tout cela a donné à la pièce un caractère temporel surréaliste.

En effet, le temps est un vrai défi qui occupe une place particulière dans le théâtre de Salacrou, celui-ci est le premier à nommer les actes par "tableaux", raison pour laquelle il est considéré comme le précurseur de la scénographie temporelle moderne.

Ulysse, personnage principal, échappe à son présent en se suicidant mais le temps le piège en le faisant revivre son passé. II se rattache ainsi de nouveau à sa vie passée, se réjouit même de la revivre mais la magie du temps a toujours un prix et ses aspirations ne sont que dérisoires. Nicolas, son valet et maître du jeu du temps repris, lui rappelle que la balle qu'il s'est tirée a déjà fait son chemin et que sa mort est inévitable : "Hélas ! Le coup est déjà parti et rien au monde ne l'empêchera d'arriver »" Cette structure fractionnelle du temps offre une liaison des images qui sont en effet attachées d'une façon illogique les unes aux

12 Armand SALACROU, L'Inconnue d'Arras, in Théâtre III, Paris, Gallimard, nrf, 1942, p. 214 . 
autres mais qui semblent plutôt enchaînées par l'imagination d'un mort dont l'inconscient émerge d'un coup devant les spectateurs. Dans cette pièce, le dramaturge met en lumière la question dont il n'avait pas auparavant perçu l'essence : le temps est-il réalité ou illusion ? La vie est-elle un simulacre, un semblant d'être, pour ne pas dire un non-être ? Et la réponse de Salacrou est que la réalité n'est simplement qu'une illusion au niveau existentiel comme au niveau temporel insinuant une lamentable parodie irrémédiable, ce qui enfonce le personnage dans le remords : "Plus tard, ratatiné, se souvenant de sa jeunesse, on ose dire, comme un miteux, comme un cafard : "j'avais tort" ${ }^{13}$. Ces mots d'Ulysse le confinent dans un espace qui lui permet d'échapper à sa réalité intérieure et extérieure en se réfugiant dans les rêves : "oublié et délaissé " ${ }^{14}$, se lamente-t-il.

Précisons que Salacrou modernise les conceptions de Maeterlinck concernant l'absence de I'homme en faveur d' " une projection de formes symboliques, par un être qui aurait les allures de la vie sans avoir la vie ${ }^{15}$. Au fait, nous pouvons

13 L'Inconnue d'Arras, p.151.

${ }^{14}$ Aurélie Diane HAROCHE, Réflexions sur le théâtre de Jean Anouilh et d'Armand Salacrou et l'évolution de leur réception, Thèse, Paris IV, 2006, p. 111.

15 Jacqueline de JOMARON, La Mise en scène contemporaine // (1914-1940), Paris, Armand Colin, 1992, p. 213. 
constater qu'Ulysse n'est pas un personnage au sens usuel du terme. II n'est pas présenté de l'extérieur, mais son créateur nous emmène dans son subconscient et même dans son inconscient.

Le rideau se lève avec le retentissement du coup de revolver annonçant le suicide d'Ulysse. Sa femme se précipite en larmes, accompagnée du valet Nicolas. Le grand voyage au milieu des souvenirs doit permettre d'élucider les raisons du suicide et donner un sens à une existence qui vient de s'achever. Comme Ulysse est désormais un homme exterminé qui n'a plus de futur, la notion du temps est forcément embrouillée.

Le nouveau-mort est d'abord confronté à réécouter tous les propos qu'il a jadis entendus au cours de sa vie, puis à ceux qu'il a lui-même prononcés. Ensuite, toutes les personnes qu'il a rencontrées et qui peuplaient sa mémoire ressurgissent devant lui et lui parlent pour une dernière fois. Ulysse revoit ainsi toute sa vie jusqu'au suicide par lequel la pièce se termine comme elle a commencé. Les personnes avec qui il aurait aimé passer le temps ne font pas partie des vivants, l'indice de l'irrévocabilité s'imposant :

«Le Grand-Père : Je t’aime, mon petit. 
Ulysse: Je le sais, mais vous n'êtes qu'un portrait. Je pense à vous souvent, je sais que vous m'auriez aimé. Mais vous n'êtes pas près de moi sur la terre. Je ne pourrai vous rencontrer que plus tard au rendez-vous que la Mort donne aux vivants " ${ }^{16}$. Peu avant la tombée du rideau, il cherche à révoquer son action désabusée mais trop tard puisque les jeux sont effectivement faits. Face à son impuissance et au temps figé qui l'enferme, il adopte la solution la plus passive, celle de ne rien faire, de croiser les bras et d'attendre puisqu'en tout cas, il ne pourra jamais modifier son destin. Nicolas n'arrive pas à le consoler, au contraire, il le provoque : «Mais est-ce vivre que de vivre comme vous avez vécu ? Comme vivent tous les hommes...les mains tendues vers les minutes qui naissent... vers l'heure qui vient...les mains tremblantes au bord du jour qui se lève. Essayant de deviner, comme un aveugle une destinée perpétuellement naissante ? "17

La dualité et la fuite perpétuelle du temps sont les procédés fondamentaux du théâtre salacrien pavant déjà la route au Nouveau Théâtre et à la révolution scénographique. Lucie dans la pièce Atlas-Hôtelappréhende la fuite du temps au milieu d'une

\footnotetext{
${ }^{16}$ L'Inconnue d'Arras, p.191.

17 Ibid., pp. $128-129$.
} 
famille où "les générations se mêlent vite ${ }^{18}$ et dont les membres ne font qu'assister à des mariages et des baptêmes pour accrocher les photos de ces moments sur le mur, des moments qui ne vont jamais revenir, façon donc d'essayer de figer le temps avec ces photos qui ne font que jaunir. Lucie refuse de subir le même sort d'Albany qui se repentit : «Hélas ! Je ne croyais pas à vingt-cinq ans, que la vie fut si courte, et que l'on arrivait si vite aux cheveux blancs - et que ce que l'on payait du prix de son bonheur, de l'orgueil de soi-même, de son âme, fut si fragile et si éphémère ${ }^{19}$

Milan Bunjevac souligne qu' "il y a là comme un souvenir : le sujet vit la scène sans être submergé ni déçu par elle " ${ }^{20}$. Le temps présent et le temps passé sont confondus dans le même plan, le retour en arrière est indiqué par un glissement imperceptible, équivoque et illimité et non par le procédé de coupure franche : "Ulysse commence par la fin, retourne dans le passé, revient au présent, [...] revoit une scène de sa vie, revient à nouveau au dernier jour de son existence... Ainsi le

\footnotetext{
${ }^{18}$ Armand, SALACROU : Atlas-Hôtel, in Théâtre II, Paris, Gallimard, nrf, (édition définitive), 1978 , p. 143.

19 Ibid., p. 122.

20 Milan BUNJEVAC, "Le problème du temps dans L'inconnue d'Arras d'Armand Salacrou » in Travaux de linguistique et de littérature, Paris VIII, n² 2, 1970, p.129.
}

\section{4}


retour en arrière se décompose-t-il [...] en toute une suite d'épisodes qui représentent des retours en arrière partiels mais assez isolés et repliés sur eux-mêmes.» ${ }^{21}$.

La confrontation avec les différentes femmes qu'il a connues dans sa vie - un des passages les plus réussis d'une œuvre aujourd'hui en partie tombée dans l'oubli - n'est en fait que la preuve de son échec dans ses liaisons amoureuses, de ses désillusions et mensonges.

Ulysse a découvert trop tard, en revoyant sa vie, la valeur des petits moments qu'on gaspille et qu'on ne peut jamais récupérer. II constate alors que le temps nous échappe et nous fait regretter l'irréparable : «Ce qui m'a frappé, c'est le nombre de minutes et d'heures perdues. Minutes d'attentes, comme elles sont nombreuses [...] ; il est deux heures moins cinq...on attend deux heures ! Cinq minutes ou il ne se passe rien, que d'attendre deux heures... On attend une femme, on attend l'autobus, on attend le départ d'un train, $[\ldots] »^{22}$. Soulignons que la valeur de ces moments ne peut être appréciée que par un mort, puisqu'il en a été privé. La fuite du temps est ainsi une source de souffrance pour le personnage puisqu'elle lui annonce la mort inintelligible

${ }^{21}$ Ibid. p.139.

22 Ibid. p.148. 
et la dualité du temps impose « une sorte de prise de conscience tragique chez le héros impuissant devant son passé irrécupérable $\eta^{23}$. Les pièces de Salacrou exposent donc une temporalité compliquée des réalités : «fabrique de sens, fabrique de l'image, enjeu politique, lieu de quête personnelle de l'acteur, laboratoire inépuisable de formes, croisement du passé et du présent ${ }^{24}$.

Le dramaturge fait appel à un autre procédé temporel dans L'Inconnue d'Arras. Après un retour en arrière agité, la pièce se termine par un effet statico, avec la mort comme réponse finale à toutes les tentatives des protagonistes. La pièce a commencé et s'est achevée par un coup de la même arme, comme si tout ce qui s'était passé au milieu des deux coups n'a duré que le temps de la déflagration de la balle tirée. Le deuxième coup a mis fin aux moments infernaux des souvenirs d'un mort trahi par sa femme. C'est ainsi que le refuge dans le passé ne réussit jamais. Le personnage reste déçu par la fuite du temps, par la

\footnotetext{
${ }^{23}$ Hani DANIEL, Le théâtre de Salacrou face aux réalités du monde contemporain, Thèse de Doctorat, Université d'Ain-Shams, Le Caire, 1992, p. 252.

[Bibliothèque Nationale de France, Notice $n^{\circ}$ FRBNF37161858;

http://catalogue.bnf.fr//ark:/12148/cb37161858h/PUBLIC]

${ }^{24}$ Christian BIET et Christophe TRIAU, Qu'est-ce que le théâtre ? Gallimard, coll. «Folio Essais », Paris, 2006, pp. 716-717.
} 
fatalité, mais continue à rêver de revivre les moments passés, du " temps que nous ne pouvons attraper que par la queue. " 25

Le temps, passé ou futur, est en effet mis à mort ; il ne reste plus que le moment présent qui s'avère certain car il se définit par l'autre certitude absolue qu'est la mort.

La fuite du temps n'est donc pas un mal en soi ; sa problématique réside dans le fait qu'elle mène à la mort, à l'irrémédiable trou noir.

De là, Salacrou insiste sur l'importance du souvenir pour ce personnage obligé à revivre son passé. Cette importance est expliquée par l'auteur dans une phrase qu'il a empruntée à Unamuno: "Pour avoir beaucoup d'espoir, il faut avoir beaucoup de souvenirs ${ }^{26}$. A son tour, Ulysse, quoique torturé par ses souvenirs, avoue : "Sur la terre, un homme sans souvenirs est un homme perdu. " ${ }^{27}$

Une fois de plus Salacrou a recours au procédé de la dualité du temps pour faire revivre des moments que ses personnages n'ont pas su vivre lorsqu'ils leur ont été offerts.

Aussi Ulysse a-t-il recours au grenier de ses souvenirs d'enfance et de jeunesse éprouvant le besoin de revivre une

\footnotetext{
${ }^{25}$ Jean-Paul, SARTRE, La Nausée, Paris, Gallimard, collec. Folio, 1938, p. 64.

${ }^{26}$ Armand SALACROU, Théâtre III, op.cit., p.217.

${ }^{27}$ L'Inconnue d'Arras, p. 213.
} 
pureté non acquise au long des années. Le recours des personnages salacriens au temps rétrospectif est donc un moyen de trouver leur salut, en essayant de se rattraper, de réparer les maladresses jadis commises, tout en étant pourtant sûrs de l'inefficacité et la vacuité de ce procédé. Nicolas l'affirme : "Dans la vie tout est unique: les expériences comme les secondes qui passent. Un remords ne déplace pas un geste. Un regret n'efface pas une parole ${ }^{28}$. Ulysse essaie de se défendre, il répond alors laconiquement : «Parce que j'ai suivi la mode de mon temps ${ }^{29}$.

Le même personnage nous est présenté à des âges différents de sa vie comme le cas de Maxime 20 ans et Maxime 37 ans ; il apparait physiquement en deux images fictive et réelle, celle du passé et celle du présent, défilant sur scène en même temps : «Le passé redevient présent sans cesser pourtant d'être organisé comme un souvenir : Le sujet vit la scène sans ne être submergé ni déçu par elle. " ${ }^{30}$ Le présent et le passé sont confondus dans le même plan, le retour en arrière n'est pratiquement pas possible. Tout se noue telle une ronde qui

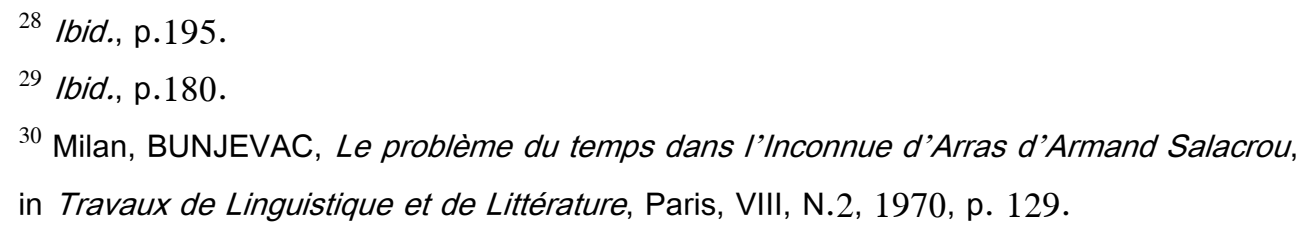


tourne et l'on a l'impression qu'il n'y a plus de début ni de fin. Yvette a raison de constater : «Nous ne vivons plus à la file des jours. Nous tournons en rond. " ${ }^{31}$

Quant à Jeanne de Comme les Chardons, sa position dans ces retours au passé est celle de spectatrice. II lui arrive une ou deux fois d'entrer dans le jeu et de se mettre à parler comme si elle se trouvait de nouveau dans ce passé ; mais en réalité, sa position n'est que celle de l'observatrice/commentatrice. Son personnage est parfois doublé par celui de Jeanne jeune. Elle se regarde donc parler et agir, mais comme s'il s'agissait de quelqu'un d'autre, sans s'identifier avec elle-même à l'âge jeune. Elle finira alors par s'écrier : «Le passé, c'est l'enfer ! " ${ }^{32}$ Le dramaturge montre combien le temps est inexorable et que l'espoir en une "deuxième chance" susceptible de compenser les ratages antérieurs est hypothétique. Les contradictions flagrantes de la condition humaine s'expriment, chez lui, par l'omniprésence de la gaieté, par la raison ainsi que par l'amertume. Et si l'humour occupe une place primordiale au sein de ce théâtre c'est qu'il s'agit d'un humour plutôt noir. Tout au long des situations désespérées, les protagonistes salacriens «simulent des

${ }^{31}$ L'Inconnue d'Arras, p. 199.

${ }^{32}$ Armand, SALACROU, Comme les Chardons, Paris, Gallimard, collection Folio, 1956, p. 349. 
grimaces avant de contempler la réalité. Ce n'est qu'après l'apparition d'un effet comique spontané, créé par l'absurdité de la situation [...], que Salacrou dévoile le masque pour découvrir un miroir déformé ${ }^{33}$.

D'ailleurs, le dramaturge ne se contente pas de pratiquer, sur scène, le retour en arrière d'inspiration cinématographique mais use aussi du procédé du théâtre dans le théâtre.

Les indications scéniques, le découpage des actes et le décor schématisé - réduit à sa plus simple expression - permettent tous ces agencements du temps de façon à ce que les personnages deviennent en effet des personnages-spectateurs. Ils sont le premier public et jouent pour eux-mêmes la comédie de la vie qu'ils ont menée.

Nicolas, le valet dans L'Inconnue d'Arras, annonce : «Monsieur, tout est là ! Toute votre vie ! Les hommes, les femmes, les meubles, les gosses, les livres, le bric-à-brac et la bricole, depuis la sage-femme jusqu'à l'armurier ! „34

Ce théâtre dans le théâtre favorise de même la dualité du temps rétrospectif : Le grand-père d'Ulysse, jeune soldat tué à Gravelotte à vingt ans peu de temps après avoir appris que sa

\footnotetext{
${ }^{33}$ Yvonne Thérèse ELIANE, L'Angoisse derrière le masque grinçant : Salacrou et le théâtre de l'humour noir, thèse, McMaster University, Hamilton, Ontario, Octobre 1971, p.43.

${ }^{34}$ Ibid., p.140.
} 
femme était enceinte, s'effondre quand il découvre que le bébé dont il rêvait et que la mort l'en a privé n'est actuellement qu'un vieillard : «Oui, ce vieillard, c'est votre espoir de petite fille ! "35, lui rétorque La Mère Venot. Le personnage reste sidéré sous le choc de la dualité du temps qui l'écrase.

Quant à "I'Inconnue", jeune fille qu'Ulysse officier aviateur a rencontrée dans les ruines de la ville au cours de la première guerre mondiale, elle intervient pour lui rappeler les détails de leur rencontre sans pour autant déceler le mystère qui l'a depuis toujours enveloppée. Même si elle a acquis une extrême importance parmi les souvenirs vivants du moment, elle demeure le symbole d'une rencontre inaboutie qui aurait pu changer la vie d'Ulysse mais hélas, le présent, quoique fictif, est la seule réalité : "C'est toujours le présent qui est concerné, et tente désespérément de se saisir depuis l'évocation d'un passé jamais

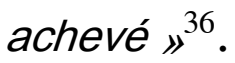

Le procédé du théâtre dans le théâtre s'avère commode pour l'auteur puisqu'il nourrit ses intentions mais révèle, paradoxalement, l'inanité des tentatives rétrospectives de justification faites par les personnages qui n'apprécient pas le

35 Ibid., p.148.

${ }^{36}$ Dominique VIART \& Bruno VERCIER, La Littérature française au présent, Paris, Bordas, 2005, pp.85-86. 
temps de leur vivant et le gaspillent. Ulysse le reproche sans aménité à son père : "Tiens, dans une vie aussi longue que la tienne, je suis sûr que les seules minutes perdues réunies permettraient de loger une autre destinée humaine, plus courte mais peut-être fort brillant " ${ }^{37}$.

Or si vaines que soient nos motivations, nos actes n'en sont pas moins irréversibles : "Ulysse : pourquoi m'as-tu trompé ? Yolande : Mais je ne sais pas. Je ne sais plus " ${ }^{38}$. A la fin de la pièce, Ulysse, après avoir revécu, mais surtout "rejoué" sa vie, ne veut plus mourir, dans l'espoir de réparer cette existence ratée, de la mieux revivre ; mais l'évidence n'est pas la scène théâtrale et le théâtre dans le théâtre se disloque face à la réalité humaine : "Dans la vie tout est unique : les expériences comme les secondes qui passent. Un remords ne déplace pas un geste. Un regret n'efface pas une parole. Ce qui a été dit sera répété "39. L'impuissance du personnage ne fait que s'aggraver face au temps irrévocable et irrémédiable qu'il ne peut hélas maîtriser. Régine Robin confirme cette idée : «Le passé n'est pas libre. //

\footnotetext{
37 L'Inconnue, p.148.

38 Ibid., p.200.

39 Ibid., p.195.
} 
est régi, généré, conservé, raconté, commémoré ou haï. Qu'il soit célèbre ou occulte, il reste un jeu fondamental du présent» ${ }^{40}$.

Hanté par ses mémoires, par le piège de la dualité du temps que Salacrou expose par excellence, le personnage qui ne décide ni de son passé irréversible ni de son avenir mystérieux et qui n'est qu'un témoin désemparé, sans confiance en toute valeur humaine, en proie à un monde plein de mal et d'injustice, tente de dénicher un équilibre moral et surtout d'affronter les préjugés dont il a beaucoup souffert, le temps qui a fait bouillonner ses regrets et les misères ineffaçablement attachées à sa mémoire mais la seule solution qui lui est offerte est d'observer sa vie passée sans pourtant avoir le pouvoir de la modifier.

Malgré la dualité du temps qui tourbillonne ses personnages, Salacrou insiste sur l'impossibilité de communication efficace avec le passé. II développe un style analogue au thème de l'aliénation temporelle servant à parfaire ses techniques scénographiques, à savoir les retours en arrière et les petites séquences temporelles.

Rêveur de pureté, d'un rôle positif à accomplir dans la vie, il n'a pu, tout comme ses personnages, contrôler le temps et par suite

\footnotetext{
${ }^{40}$ Régine ROBIN, La mémoire saturée, Paris, Stock, collection « Un Ordre d'Idées », 2003, p.27.
} 
n'a pu réaliser ses rêves. Pour lui, le présent restera enchaîné par la fatalité d'un passé immuable et perdu, le tout menant à un futur énigmatique qui ne promet que la décrépitude et la mort. Les protagonistes ne sont ainsi sûrs que de leur effacement total. Le retour au passé ne constitue donc pour ces personnages qu'une tentative inutile d'oublier le présent, une sorte d'anesthésie. En réalité, ce retour est une arme à double tranchant; au lieu de réconforter le personnage il augmente sa souffrance car si le passé renferme de beaux souvenirs, il en a également de fâcheux. Le personnage se trouve alors en lutte contre le désir d'effacer une part de son passé et d'en conserver une autre mais le tri n'est point possible, l'impuissance de nier ou d'oublier s'imposant : «Oublier, quel enfantillage !» ${ }^{41}$, comme dirait Inès de Huis Clos de Sartre. Adrienne de La Femme Libre est dans le même "enfer". Elle a renié son présent et son avenir en choisissant de se réfugier dans la mort spirituelle, mais elle ne peut rien contre son passé sur quoi elle est moins puissante qu'elle l'est sur son présent et son avenir. Ce qui est fait n'est plus à refaire. Elle résume sa situation : «Les morts sont enfermés dans leur souvenirs, plus étroitement que dans leur cachot. [...], dans l'étouffement de leurs souvenirs qui sont 
définitifs. Moi, je suis morte, et les morts, sans avenir, sont terriblement immobiles. Les morts ne peuvent ni modifier la place de leurs os ni changer de souvenirs. Le passé est un enfer dont les morts ne peuvent plus sortir. " ${ }^{42}$

Le théâtre de Salacrou demeurera le théâtre statico où le temps répété et figé dévore tout espoir et ne mène qu'à la mort certaine. La dualité du temps y est donc valorisée par un mort comme Ulysse, une désespérée comme Lucie ou une personne passive comme Jeanne. Ils sont les seuls à pouvoir estimer la valeur du temps, les moments perdus et les moments présents de la vie dont ils ont été privés à cause de leur inquiétude de l'avenir et de leur regret envers ce qui n'est plus récupérable.

Et c'est ainsi que Salacrou nous initie, par la voie même du regret, à s'attacher au pouvoir du présent et à prendre conscience de chaque moment vécu qui compte être la seule force qu'un être humain puisse posséder.

\footnotetext{
${ }^{42}$ Armand, SALACROU, Une Femme Libre, in Théâtre III, Paris, Gallimard, nrf, 1942, pp. $100-101$.
} 


\section{BIBLIOGRAPHIE}

\section{EEuvres de Salacrou}

- L'Inconnue d'Arras, in Théâtre III, Paris, Gallimard, nrf, 1942.

- Une Femme Libre, in Théâtre III, Paris, Gallimard, nrf, 1942

- Le Casseur d'assiettes, in Théâtre I, Paris, Gallimard, nrf, 1943.

- Mes certitudes et incertitudes in Théâtre VI, Paris, Gallimard, nrf, 1954.

- Comme les Chardons, Paris, Gallimard, collection Folio, 1956.

- Une Femme trop Honnête, Paris, Gallimard, collection Folio, 1956.

- Le Miroir, in Théâtre VII, Paris, Gallimard. nrf, 1956.

- Atlas-Hôtel, in Théâtre I/, Paris, Gallimard, nrf, (édition définitive), 1978.

\section{Autres œuvres}

- Jean-Paul SARTRE, La Nausée, Paris, Gallimard, collection Folio, 1938.

- Jean-Paul SARTRE, Huis Clos, Paris, Gallimard, 1947.

\section{Ouvrages de critique, thèses et théories}

- Tomasz, KACZMAREK, Entre le temps de Kant et le temps D'Einstein, ou requête pour un texte expressionniste français, études romanes de BRNO, 2010.

- Christian BIET et Christophe TRIAU, Qu'est-ce que le théâtre ? Gallimard, collection «Folio Essais », Paris, 2006.

- Hani DANIEL, Le théâtre de Salacrou face aux réalités du monde contemporain, Thèse de Doctorat, Univ. D’Ain-Shams, Le Caire, 1992. [Bibliothèque Nationale de France, Notice $n^{\circ}$ : FRBNF37161858 :

http://catalogue.bnf.fr//ark:/12148/cb37161858h/PUBLIC]

- Jacqueline DE JOMARON, La Mise en scène contemporaine // (19141940), Paris, Armand Colin, 1992.

- Fiorenza Di FRANCO, Le Théâtre de Salacrou, Paris, Gallimard, 2011. 
- Yvonne Thérèse ELIANE, L'Angoisse derrière le masque grinçant : Salacrou et le théâtre de l'humour noir, thèse, McMaster University, Hamilton, Ontario, Octobre 1971.

- Marcel GABRIEL, Singularité d'Armand Salacrou, Théâtre, $3^{\text {ème }}$ cahier. (Aspects du théâtre contemporain en France), Paris, Albin Michel, 1947.

- Jerzy GROTOWSKI, Vers un théâtre pauvre, éd. L'Âge d'Homme, Lausanne, 2002.

- Aurélie Diane HAROCHE, Réflexions sur le théâtre de Jean Anouilh et d'Armand Salacrou et l'évolution de leur réception, Thèse, Paris IV, 2006.

- Henri-René LENORMAND, Les Confessions d'un auteur dramatique 2, Paris, Albin Michel, 1953.

- Régine ROBIN, La mémoire saturée, Paris, Stock, collection «Un Ordre d'Idées », 2003.

- Pascale ROGER, La Cruauté et le théâtre de Strindberg, Paris, L'Harmattan, 2004.

- Léa VALETTE, Histoire des arts et des représentations. Les lieux de la critique de théâtre en France : Enjeux esthétiques et convictions politiques, thèse, Université Paris-Ouest Nanterre, novembre 2014.

- Dominique VIART \& Bruno VERCIER, La Littérature française au présent, Paris, Bordas, 2005.

\section{Articles de périodiques}

- Milan BUNJEVAC, «Le problème du temps dans L'inconnue d'Arras d'Armand Salacrou » in Travaux de linguistique et de littérature, Paris VIII, $\mathrm{n}^{\circ} 2,1970$.

- John WHEELER, «Nécrologie », in Le Monde, $\mathrm{n}^{\circ}$ 19669, 20-21 avril 2008. 
- Roland BARTHES, Introduction à l'analyse structurale des récits, article, recherche sémiologique, 1966.

- Solenne ARNAUD, La désorientation spatio-temporelle chez la personne âgée ayant la maladie d'Alzheimer : l'apport de la psychomotricité dans la prise en charge des troubles psycho-comportementaux, archive-ouvertes.fr, Psychologie. 2019.

- John BARGH, La puissance de l'inconscient, Pour la Science n. 437, février 2014.

- Daniel, MAGETTI, Etude du personnage sacrificiel dans quatre œuvres romanesques de Maurice ZERMATTEN : Christine, Le Jardin des Oliviers, La Fontaine d'Aréthuse, Le Bouclier d'or, Université de Lausanne, juin 2007, France.

- David, LOPEZ, Les troubles du comportement : une maladie de la culture qui fait symptôme dans le lien social et interroge l'École, Université Paul Valéry - Montpellier III, 2017.

\section{Dictionnaires}

- PAVIS, Patrice, Dictionnaire du théâtre, Paris, Armand Colin, 2002.

\section{Sites consultés :}

file://C:/Users/Marguerite/Downloads/Dialnet-

EntreLeTempsDeKantEtLeTempsDEinsteinOuRequetePourU$4363783 \% 20(1) \cdot p d f$

https://www.cairn.info/l-esthetique-du-tableau-dans-le-theatre-du-xviii-9782130495390-page-153.htm

https://fr.wikisource.org/wiki/Page:Bergson_L L\%E2\%80\%99\%C3\%89voluti on_cr\%C3\%A9atrice.djvu/349 
http://expositions.bnf.fr/homere/arret/10.htm

https://digilib.phil.muni.cz/bitstream/handle/11222.digilib/113539/1_Etudes RomanesDeBrno_02-1966-1_11.pdf?sequence=

https://tel.archivesouvertes.fr/file/index/docid/656759/filename/Viollet_Mari on.pdf

http://www.acgrenoble.fr/PhiloSophie/logphil/notions/interpre/esp_prof/synt hese/reves.h 